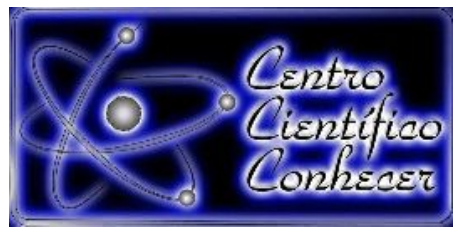

\title{
AVALIAČ̃̃ DAS POTENCIALIDADES PARA A AGRICULTURA URBANA NO ENTORNO DA UNIVERSIDADE FEDERAL RURAL DA AMAZÔNIA (UFRA), MUNICÍPIO DE BELÉM (PA)
}

Laila Brabo Pacheco ${ }^{1}$; Fabrício Khoury Rebello²; Marcos Antônio Souza dos Santos ${ }^{3}$; Paulo Silvano Magno Fróes Júnior ${ }^{4}$; Silvana Meireles da Silva ${ }^{5}$

1. Bolsista PIBIC/FAPESPA, Estudante de Agronomia da Universidade Federal Rural da Amazônia (UFRA). Belém, Pará.

2. Professor do Programa de Pós-Graduação em Agronomia (PGAGRO), Universidade Federal Rural da Amazônia (UFRA). Belém, Pará. E-mail: fabriciorebello@hotmail.com.

3. Professor do Programa de Pós-Graduação em Agronomia (PGAGRO), Universidade Federal Rural da Amazônia (UFRA). Belém, Pará.

4. Mestrando do Programa de Pós-Graduação em Agronomia (PGAGRO/UFRA). Belém, Pará.

5. Estudante do último semestre do Curso de Agronomia da Universidade Federal Rural da Amazônia (UFRA). Belém, Pará.

Recebido em: 19/11/2018 - Aprovado em: 14/12/2018 - Publicado em: 25/12/2018 DOI: 10.18677/Agrarian_Academy_2018B3

A agricultura urbana (AU) é um conceito multidimensional de produção agrícola que vem ganhando espaço na economia dos centros urbanos. Sua importância se dá pela produção de alimentos para o autoconsumo e segurança alimentar, além de possibilitar a venda a partir de um canal de comercialização mais curto, aproximando o produtor do consumidor. A presente pesquisa foi desenvolvida no bairro de Montese, localizado na periferia do município de Belém, capital do estado do Pará (PA), onde se encontra localizada a Universidade Federal Rural da Amazônia (UFRA). O propósito do estudo foi analisar o potencial da AU no bairro. Para tanto foram realizadas entrevistas junto a 40 moradores do bairro, no período de abril a agosto de 2018, com vistas a levantar suas condições de vida e as práticas de agricultura realizada na localidade. Constatou-se que $42,5 \%$ dos entrevistados praticam a $\mathrm{AU}$, ainda que de forma incipiente, destinando sua produção, principalmente, para satisfazer pequenas necessidades de consumo e preparo de remédios caseiros. Segundo os moradores, a maior limitação para 0 desenvolvimento da AU no bairro é a carência de orientação técnica e de incentivo. A atividade na área de estudo pode ser estimulada a partir de estratégias de extensão universitária, como forma de proporcionar apoio técnico de qualidade a esses moradores. Isso, certamente, vai demandar a articulação de uma ampla parceria envolvendo lideranças da comunidade, prefeitura, secretarias de agricultura e urbanismo, igrejas, universidades, entre outros atores, como forma de buscar encaminhamentos práticos para superar demandas técnicas, logísticas e de infraestrutura.

PALAVRAS-CHAVE: Bairro de Montese. Desenvolvimento local. Meio ambiente urbano. 


\title{
EVALUATION OF POTENTIALITIES FOR URBAN AGRICULTURE AROUND THE UNIVERSIDADE FEDERAL RURAL DA AMAZÔNIA (UFRA), IN THE MUNICIPALITY OF BELÉM (PA)
}

\begin{abstract}
The Urban Agriculture (UA) is a multidimensional concept of agricultural production that has been gaining space in the economy of urban centers. Its importance is given by the production of food to the self-consume and food security, beyond the possibility to commercialization in a marketing channel shorter that approximate the producer to the consumer. The present research was developed at the Montese Neighborhood, situated in the periphery of Belém City, capital of Pará State (PA), where is located the Universidade Federal Rural da Amazônia (UFRA). The propose of this study was analyze the potential of UA at the Neighborhood. For that were applied 40 interviews to the residents of the Neighborhood, in the period from April to August of 2018 , with the objective of obtain data about the conditions of life and about the practices of agriculture performed in the location. Thus, was find out that $42.5 \%$ of the interviewers practices the UA, even it still has been incipient, it destines its production, mainly to satisfy small necessities of consume and prepare of home remedies. According with the residents, the major limitation to the development of UA at the Neighborhood is the lack of technical guidance and incentive. The fortification of this activity in the studied area could be initiated since from strategies of University extension to proportionate technical support with quality to these residents. This will demand the articulation of an ample partnership involving community leaderships, prefecture, secretary of agriculture and urbanism, churches, universities and other actors, as a way of bringing practical directions to achieve the technical demands, logistics and of infrastructure.
\end{abstract}

KEYWORDS: Local development. Montese neighborhood. Urban environment.

\section{INTRODUÇÃO}

Em todo o mundo, o desenvolvimento dos países está sendo afetado por um processo acelerado e não planejado de urbanização agravado pela recessão econômica, aumento nos preços dos alimentos e pelos impactos da mudança climática. Os efeitos combinados desses fenômenos prejudicam a possibilidade de alcance de um desenvolvimento sustentável e equitativo (FAO/MDS, 2010).

$\mathrm{Na}$ América Latina, a expansão urbana tem gerado uma crescente insegurança alimentar nas cidades e na sua periferia, particularmente, entre os setores populacionais vítimas da pobreza e da extrema pobreza (FAO/MDS, 2010). Existe também a questão do inchaço populacional das áreas urbanas que é causado pelo processo de migração de pessoas muitas vezes advindas de regiões socioeconomicamente abaladas por diversos fatores de cunho ambiental, político, empregatício, entre outros, e que em vários casos são indivíduos que possuem baixa qualificação profissional para as atividades urbanas (REBELLO, HOMMA, 2017).

Essas questões promovem um efeito problemático nas cidades relacionado à fome, saúde, segurança pública, educação e a empregabilidade, além de vários outros impasses. Neste contexto, a Agricultura Urbana e Periurbana (AUP) aparecem como uma oportunidade de saída democrática, participativa e construtora da cidadania, que necessita ser promovida e apoiada tanto pelo poder público como pelas organizações da sociedade civil preocupadas com um futuro melhor para o Brasil (SANTANDREU; LOVO, 2007; FAO/MDS, 2010). 
A AUP é entendida como um conceito multidimensional que inclui a produção, transformação e a prestação de serviços, de forma segura, para gerar produtos agrícolas e pecuários voltados ao autoconsumo, trocas e doações ou comercialização, (re)aproveitando-se, de forma eficiente e sustentável, os recursos e os insumos locais (SANTANDREU; LOVO, 2007).

É importante destacar que nesse modelo de atividade, visto à inserção aos centros urbanos, percebe-se potencialidades de redução de custos com logística referente ao estreitamento dos canais de comercialização, ou seja, o agricultor e o consumidor tem a possibilidade de se relacionarem mais diretamente devido à proximidade entre os dois atores (SANTANDREU; LOVO, 2007; FAO/MDS, 2011).

Segundo Ferreira et al. (2018) a Agricultura Urbana suporta a promessa da segurança alimentar e de supremacia dos alimentos, tendo a perspectiva de crescimento, por meio da contribuição de uma economia de baixa emissão de carbono como resultado da cadeia de suprimentos mais curta e com menor uso de combustíveis fósseis no transporte.

Para que a AUP possa expandir é necessário ter um conhecimento maior dessa prática. Assim, a discussão a respeito da agricultura urbana não é de caráter local ou regional, de um estado ou país, as pesquisas demonstram que cada vez mais populações urbanas investem na produção de alimentos dentro dos espaços urbanos (SILVA; SABLAYROLLES, 2015).

Segundo Boukharaeva et al. (2005), na França, o aparente interesse e a boa vontade dos poderes públicos foram associados a uma política fundiária urbana que não parou de demarcar os terrenos disponíveis. No caso da Rússia e de outros países da ex-União Soviética mostram que as tendências a sua marginalização, constatadas igualmente nos países ocidentais, não são uma lei irreversível da modernidade. No Brasil, onde a luta contra a fome e pela promoção das populações pobres é apontada como prioridade nacional, as condições estão, talvez, reunidas para que a agricultura urbana seja reconhecida no mesmo nível da agricultura rural, da industrial ou dos serviços, e seja explicitamente considerada nos planejamentos urbanos, nas políticas alimentares, sanitárias e educativas.

É interessante ter a percepção de que a maioria das iniciativas de agricultura urbana são, em geral, reivindicações sociais. Sua demanda por espaço nas cidades confronta com os diversos usos que uma cidade pode oferecer, e em se tratando de produção agrícola, faz-se necessário um espaço concreto, pela dependência de solo, e que somente torna-se vistosa aos olhos do estado se está atrelada aos meios econômico e social (ROSA, 2011). Porém, de acordo com Schneider e Xavies (2013), os dados qualitativos sobre este tipo de agricultura ainda são escassos no Brasil, o que dificulta a formulação e implementação de políticas.

Acredita-se que essa atividade é um importante mecanismo econômico, social e ambiental para a concretização das "cidades do futuro", onde o relacionamento entre a produção vegetal e pecuária e os centros urbanos são mais estreitos, o que contribui para o desenvolvimento socioeconômico sustentável, além de promover a sensação de bem-estar por causa da proximidade do homem com a natureza (SANTANDREU; LOVO, 2007; FAO/MDS, 2010; FAO/MDS, 2011, DAROLT et al., 2016; COSTA et al., 2017).

Assim, nesta pesquisa, foram levantados dados sobre o perfil socioeconômico, ambiental e produtivo da área no entorno da Universidade Federal Rural da Amazônia (UFRA), no bairro de Montese, objetivando analisar o potencial da Agricultura Urbana na localidade, por meio da caracterização do perfil dos agricultores, dos sistemas de produção adotados, canais de comercialização, 
infraestrutura, organização social, nível tecnológico e dos recursos empregados nas atividades de agricultura urbana.

Estudos dessa natureza são importantes para contribuir com a formulação de políticas públicas que fortaleçam essa prática, a exemplos de ações de capacitação, infraestrutura e de sistematização de tecnologias apropriadas aos sistemas de produção a serem adotados. Neste caso em particular, pode servir de referência para orientar práticas de extensão universitária e, consecutivamente, contribuir para implementar melhorias socioeconômicas na comunidade.

\section{ÁREA DE ESTUDO}

\section{MATERIAL E MÉTODOS}

A pesquisa foi desenvolvida no Bairro de Montese (anteriormente denominado de Terra Firme) que faz parte da bacia do Tucunduba (área de aproximadamente 1.055 ha) localizada na parte sul do município de Belém, capital do estado do Pará, reconhecido como uma área de baixada. Sua população é de 61.439 habitantes (PMB, 2012), sendo o sétimo bairro mais populoso da cidade.

O bairro possui uma área urbana de 243,66 hectares e um total de 15.464 domicílios particulares urbanos e nenhum domicilio rural de acordo com dados da Prefeitura Municipal de Belém (PMB, 2012).

Este bairro congrega as áreas da Universidade Federal Rural da Amazônia (UFRA), Universidade Federal do Pará (UFPA), Museu Paraense Emílio Goeldi (MPEG) e Empresa Brasileira de Pesquisa Agropecuária (EMBRAPA). O bairro foi estruturado em uma localização de baixa topografia e abrange igarapés e braços de rio; características que tiveram grande influência sobre seu traçado viário e disposição das residências, exceto em áreas que fazem limite com os bairros do Marco e de Canudos (DIAS et al., 2013).

\section{BASE DE DADOS}

Para realização da pesquisa foram coletados dados primários a partir da aplicação de questionários semiestruturados com os responsáveis por domicílio do bairro de Montese, visando levantar o perfil dos agricultores urbanos e a caracterização da produção agropecuária desenvolvida.

Foram aplicados 40 questionários em diversos endereços do bairro, entre os meses de abril a agosto de 2018 durante os fins de semana e de acordo com a disponibilidade dos moradores. Os domicílios foram selecionados a partir de indicação de lideranças do bairro que apontavam contatos onde existia a iniciativa ou disposição para a prática da agricultura urbana.

As perguntas dos questionários foram divididas em três blocos, correspondendo a questões sobre o perfil socioeconômico do entrevistado (idade, sexo, escolaridade, ocupação, renda, alimentação, saúde, entre outras), caracterização da moradia (condições de infraestrutura) e um bloco específico sobre a Agricultura Urbana.

Após a coleta de dados, as informações foram tratadas a partir de técnicas de análise tabular e estatística descritiva para que pudessem gerar os resultados e discussões sobre a Agricultura Urbana praticada no bairro pesquisado.

\section{RESULTADOS E DISCUSSÕES}

\section{BREVE CARACTERIZAÇÃO DO BAIRRO}

De acordo com Silva e Sablayrolles (2015) o processo de urbanização e embelezamento do município de Belém implementado pelo intendente Antônio AGRARIAN ACADEMY, Centro Científico Conhecer - Goiânia, v.5, n.10; p.19 2018 
Lemos (1843-1913), em sua gestão entre os anos de 1887 a 1911, promoveu a transferência de muitos moradores que residiam em áreas centrais da cidade para municípios do interior e para a periferia da cidade, surgindo assim muitos bairros nas regiões mais afastadas do centro, especialmente nas áreas mais precárias em termos de saneamento e outros serviços básicos.

O bairro de Montese (Terra Firme) teve sua ocupação a partir da expansão urbana em direção às áreas de baixadas, que se intensificou em Belém, principalmente a partir da década de 1940 (SILVA; SABLAYROLLES, 2015). O nome originalmente dado ao lugar foi Terra Firme, como ironia ao fato de que no período de ocupação predominavam áreas alagadas, dificultando os ocupantes assentarem suas casas (ALCÂNTARA; GONTIJO, 2015). Em 1996, o nome do bairro foi alterado pela Prefeitura Municipal de Belém (PMB) para Montese em homenagem aos soldados brasileiros que participaram dessa batalha na II ${ }^{a}$ Guerra Mundial. Essa designação, no entanto, não pegou entre os moradores que ainda reconhecem o bairro pelo nome anterior (GOUVÊA, 2017).

No bairro predomina uma população de baixa renda vinda desse processo de urbanização do centro da cidade e de municípios do interior do estado do Pará e de estados vizinhas, como o Maranhão (LEITE JÚNIOR; ALMEIDA, 2012). Matos et al. (2011), utilizando técnicas de geoprocessamento e de visitas em campo, mostra que a área de vegetação nativa diminuiu e a urbanização aumentou, principalmente, no período entre 1972 e 2006.

\section{PERFIL SOCIOECONÔMICO DOS MORADORES}

O perfil socioeconômico dos moradores entrevistados foi avaliado a partir dos aspectos como sexo, idade (anos), grau de escolaridade, ocupação, renda familiar e recebimento de benefícios de programas sociais. Dos entrevistados, $72,5 \%$ eram mulheres e $27,5 \%$ homens. Quanto à faixa etária, 22,5\% estavam com idade entre 20 e 40 anos, $25,0 \%$ entre 40 e 60 anos e 12,5\% eram idosos com idade acima de 60 anos. Outros 40,0\% dos entrevistados não informaram a idade.

Em uma análise socioeconômica a educação é um aspecto relevante a ser considerado. No contexto educacional, a maioria (27,5\%) possuía apenas o ensino médio incompleto, seguido pelos que tinham ensino médio completo (22,5\%), ensino fundamental II (17,5\%), ensino superior incompleto (12,5\%), ensino superior $(5,0 \%)$ e não estudaram (5\%), conforme dados apresentados na Tabela 1.

TABELA 1 - Nível de escolaridade dos moradores entrevistados no Bairro de Montese, 2018.

\begin{tabular}{lr}
\hline Nível de escolaridade & Percentual (\%) \\
\hline Ensino Fundamental I (1 $1^{\underline{a}}$ a 4 ${ }^{\underline{a}}$ série) & 2,50 \\
Ensino Fundamental II (5a a 8ª série) & 17,50 \\
Ensino Médio Completo & 22,50 \\
Ensino Médio Incompleto & 27,50 \\
Ensino Superior Completo & 5,00 \\
Ensino Superior Incompleto & 12,50 \\
Analfabetos & 5,00 \\
Não respondeu & 7,50 \\
\hline Total & $\mathbf{1 0 0 , 0 0}$ \\
\hline
\end{tabular}

FONTE: dados da pesquisa. 
A educação é considerada um dos mais críticos fatores para inovação e o desenvolvimento de empreendimentos e da sociedade (REBELLO; HOMMA, 2017; DUERRENBERGER; WARNING, 2018). Dessa forma, o nível educacional de um indivíduo influência diretamente nas suas ações, na tomada de decisão, na visualização de oportunidades e no desenvolvimento humano e social.

Em muitos estudos sobre Agricultura Familiar e Agricultura Urbana, em países em desenvolvimento, é comum que o nível de escolaridade esteja entre o Fundamental I e Médio Completo. Nesse estudo foi diagnosticado que $70 \%$ dos entrevistados tinham nível educacional até o nível Médio, sendo que $17,5 \%$ tiveram algum contato com o Nível Superior e 5\% não estudaram. A presença de indivíduos com curso superior pode ser positiva para alavancar programas de treinamento quanto às práticas de agricultura urbana e de desenvolvimento local.

Objetivando levantar informações sobre a sustentabilidade financeira do grupo entrevistado, os participantes foram questionados sobre a renda mensal da família. Observou-se que $72,5 \%$ possuem renda de um a dois salários mínimos (Tabela 2). A renda de até um salário mínimo corresponde a 12,5\% e apenas 10,0\% possuem renda de três a quatro salários. Foi constatado que na composição da renda mensal $60,00 \%$ dos entrevistados tinham a colaboração de todos os membros da família.

TABELA 2 - Renda familiar mensal dos moradores do Bairro de Montese, 2018. Renda familiar mensal (S.M)

\begin{tabular}{lr}
\hline Até 1 salário & 12,50 \\
1 a 2 salários & 72,50 \\
3 a 4 salários & 10,00 \\
Não respondeu & 5,00 \\
\hline Total & $\mathbf{1 0 0 , 0 0}$ \\
\hline
\end{tabular}

FONTE: dados da pesquisa.

Nota: o Salário Mínimo (SM) vigente no período da pesquisa era de $\mathrm{R} \$ 937,00$.

Quanto à ocupação dos entrevistados, apenas 2,50\% informou trabalhar com agricultura (trabalha com horta). A maior parcela $(45,00 \%)$ possuía ocupação como pequeno comerciante, dona de casa, empregada doméstica, manicure, orientadora escolar, projetista, secretária, técnica em enfermagem, assistente ou auxiliar administrativo, professor e funcionário público. Os estudantes eram $40,00 \%$ e os aposentados somavam $5,0 \%$. Outros $7,5 \%$ não informaram sua ocupação.

Desses moradores, 32,50\% possuem Bolsa Família, 5,0\% possuem benefício pelo INSS e 7,5\% possuem aposentadoria. Dos entrevistados $32,5 \%$ informaram que não recebem nenhum tipo de benefício (Tabela 3).

TABELA 3 - Benefícios sociais recebidos pelos entrevistados, 2018.

\begin{tabular}{lr}
\hline Recebe benefício & Percentual (\%) \\
\hline Aposentadoria & 7,50 \\
Bolsa Família & 32,50 \\
INSS & 5,00 \\
Recebe, mas não especificou o benefício & 10,00 \\
Não recebe & 32,50 \\
Não informou & 12,50 \\
\hline Total & $\mathbf{1 0 0 , 0 0}$ \\
\hline
\end{tabular}

FONTE: dados da pesquisa. 


\section{CARACTERÍSTICAS DOS DOMICÍLIOS}

Quanto às moradias, procurou-se identificar as características como o tipo de construção, condição de posse e o tempo de residência, pois são indicadores importantes para aferir a qualidade de vida das pessoas que residem no bairro. A moradia padrão dos entrevistados é casa de alvenaria $(90,0 \%)$. Com relação à condição de posse, $92,5 \%$ dos moradores possuía imóvel próprio. Apenas 2,5\% disseram residir em casa cedida e $2,5 \%$ eram alugadas. Igual percentual não respondeu a essa questão.

Quanto ao tempo de residência no bairro, $72,5 \%$ moravam a mais de 10 anos e 12,5\% residem entre sete e 10 anos. Esses dados indicam que a grande maioria $(85,0 \%)$ possuía mais de sete anos de residência no bairro e que, associado à condição de proprietário do imóvel $(92,5 \%)$, respalda o interesse manifestado pelos entrevistados em buscar melhorias para implementar práticas de agricultura urbana no próprio domicílio. A Tabela 4 apresenta a estratificação completa do tempo de residência no bairro.

TABELA 4 - Tempo de residência dos moradores no bairro de Montese, 2018.

\begin{tabular}{lr}
\hline Tempo que residência & Percentual $(\%)$ \\
\hline De 1 a 3 anos & 2,50 \\
De 4 a 6 anos & 5,00 \\
De 7 a 10 anos & 12,50 \\
Acima de 10 & 72,50 \\
Não informou & 7,50 \\
\hline Total & 100,00 \\
\hline
\end{tabular}

FONTE: dados da pesquisa.

\section{SANEAMENTO, SAÚDE E ALIMENTAÇÃO}

Os entrevistados informaram que recebem água do sistema de distribuição da Companhia de Saneamento do Estado do Pará (COSANPA). Todos os moradores também são servidos por energia elétrica distribuída pelas Centrais Elétricas do Pará (CELPA). Às instalações sanitárias mais recorrentes entre os entrevistados eram as fossas sépticas $(55,00 \%)$, conforme pode ser constatado na Tabela 5 . $O$ bairro possui a coleta de lixo realizada pela prefeitura, três vezes por semana.

TABELA 5 - Tipo de instalação sanitária das moradias dos entrevistados.

\begin{tabular}{lr}
\hline Instalação sanitária & Percentual (\%) \\
\hline Rede geral & 25,00 \\
Fossa comum & 12,50 \\
Fossa séptica & 55,00 \\
Não informou & 7,50 \\
\hline Total & $\mathbf{1 0 0 , 0 0}$
\end{tabular}

FONTE: dados da pesquisa.

O bairro dispõe de Posto de Saúde localizado na Passagem São João, próximo à Avenida Perimetral. Além do posto, os moradores também utilizam o Pronto Socorro Municipal, o Hospital Universitário Bettina Ferro de Souza entre outros serviços de saúde. Os motivos mais citados para os atendimentos em saúde foram às viroses, gripes e resfriados, além disso, algumas pessoas disseram ir ao 
médico para fazer consultas preventivas. Dentre os moradores entrevistados, a minoria possui plano de saúde (20\%).

Quanto à saúde bucal, $82,5 \%$ dos entrevistados afirmaram que fazem ou já fizeram visitas ao dentista, 5,0\% afirmou que nunca foi ao dentista e 12,5\% preferiu não responder à questão. No que diz respeito à frequência de visitas ao dentista, $5,0 \%$ disseram que vão semestralmente, $15,0 \%$ anualmente, $40,0 \%$ somente quanto tem dor de dente e $20,0 \%$ afirmaram ter outras motivações para visitar o dentista, como: quando quebram o dente $(2,5 \%)$, somente quando necessita $(12,5 \%)$ e quando vai fazer limpeza (5,0\%). A questão dentária faz parte da saúde integral do indivíduo, assim tem um papel preponderante sobre as condições de trabalho e motivação geral do ser humano devendo, portanto, ser considerada no âmbito das estratégias de desenvolvimento local (REBELLO et al., 2018).

No que diz respeito ao gasto mensal com a alimentação, 37,5\% dos entrevistados informaram que desembolsam entre $R \$ 600,00$ e $R \$ 699,99$ e outros $22,5 \%$ entre $R \$ 500,00$ e $R \$ 599,99$. Apenas $5 \%$ gastam mais de $R \$ 1.000,00$ com a alimentação e 2,5\% gasta até $\mathrm{R} \$ 399,99$ (Tabela 6). Considerando uma renda média de 1,5 salários mínimos, recebido pelo estrato de maior participação na pesquisa (um a dois S.M = 72,5\%), tem-se que as famílias no estrato de gasto com alimentação de maior peso relativo ( $\mathrm{R} \$ 600,00$ a $\mathrm{R} \$ 699,99=37,5 \%)$ comprometem cerca de $46,25 \%$ de sua renda com a alimentação.

TABELA 6 - Valor mensal dos gastos com alimentação, 2018.

\begin{tabular}{|c|c|}
\hline $\begin{array}{l}\text { Valormensal de } \\
\text { alimentação (R\$) }\end{array}$ & Percentual (\%) \\
\hline Até $\mathrm{R} \$ 399,99$ & 2,50 \\
\hline$R \$ 400,00-R \$ 499,99$ & 7,50 \\
\hline$R \$ 500,00-R \$ 599,99$ & 22,50 \\
\hline$R \$ 600,00-R \$ 699,99$ & 37,50 \\
\hline$R \$ 700,00-R \$ 799,99$ & 5,00 \\
\hline$R \$ 800,00-R \$ 899,99$ & 7,50 \\
\hline Mais de $R \$ 1.000,00$ & 5,00 \\
\hline Não informou & 12,50 \\
\hline Total & 100,00 \\
\hline
\end{tabular}

Entre os entrevistados, $67,5 \%$ compram seus alimentos diariamente e $15,0 \%$ compram mensalmente (Tabela 7). Os alimentos mais citados que fazem parte da refeição básica foram: arroz, feijão, carnes (branca e vermelha), café e salada (verduras, legumes e temperos).

TABELA 7 - Frequência da compra de alimentos pelos entrevistados.

\begin{tabular}{lr}
\hline Frequência de compra dos alimentos & Percentual (\%) \\
\hline Diariamente & 67,50 \\
Semanalmente & 12,50 \\
Mensalmente & 15,00 \\
Não informou & 5,00 \\
\hline Total & $\mathbf{1 0 0 , 0 0}$ \\
\hline
\end{tabular}

Fonte: dados da pesquisa. 


\section{PRÁTICA DA AGRICULTURA URBANA}

Os entrevistados foram questionados sobre o que compreendiam sobre Agricultura Urbana. Dessa forma, definiam o termo de acordo com suas próprias ideias. As palavras-chave apresentadas nos discursos são apresentadas na Tabela 8. É interessante destacar que a grande maioria $(72,5 \%)$ apresentou alguma menção ao entendimento do conceito e $27,5 \%$ disse que não conhecia.

TABELA 8 - Definição de Agricultura Urbana pelos moradores do Bairro de Montese. Definição de AU Percentual (\%)

Plantio 37,50

Produção de Alimentos 20,00

Produção de Medicamentos 7,50

Produção no Solo (Quintais) 20,00

Produção em Vasos 25,00

Criação de Animais 10,00

Atividade Terapêutica Incremento na Renda

Não sabe dizer

Outros

FONTE: dados da pesquisa.

NOTA: Percentuais aferidos a partir de pergunta subjetiva. Sendo assim, os entrevistados puderam definir de mais de uma forma.

Dentre os entrevistados, $42,5 \%$ produziu alguma fonte de alimento dentro da sua moradia e 37,5\% não produziu (Tabela 9), mas revelou interesse em fazer.

TABELA 9 - Percentual de moradores que praticam atividades agrícolas no bairro de Montese, 2018.

\begin{tabular}{lr}
\hline Desenvolve alguma atividade agrícola no domicílio & Percentual (\%) \\
\hline Sim & 42,50 \\
Não praticam, mas tem interesse & 37,50 \\
Não informou & 20,00 \\
\hline Total & $\mathbf{1 0 0 , 0 0}$
\end{tabular}

FONTE: dados da pesquisa.

Daqueles que produzem, $27,5 \%$ desenvolvem a atividade a mais de cinco anos (Tabela 10) e somente 2,5\% produz a menos de um ano. Quanto ao local onde é exercida a prática agrícola, $35,0 \%$ informou que executa no próprio domicílio e outros 2,5\% em outro local, mas no mesmo bairro.

TABELA 10 - Tempo que prática agrícola urbana.

Tempo de prática da agricultura

Menos de 1 ano

Percentual (\%)

1 a 2 anos

2 a 5 anos

Mais de 5 anos

Não produz

Não informou 
Relacionando a participação da agricultura na renda familiar, somente 2,5\% das famílias dependem totalmente da agricultura como forma de renda (trabalha em horta) e a maior parte das famílias utilizaram menos de 10,0\% da agricultura como fonte de renda (20\%) e 7,5\% não utilizam a agricultura como forma de renda (Tabela 11).

TABELA 11 - Participação da agricultura na renda familiar.

\begin{tabular}{lr}
\hline Quanto tempo pratica a atividade? & Percentual (\%) \\
\hline Menos de $10 \%$ & 20,00 \\
De $10 \%$ a 30\% & 2,50 \\
Integralmente (100\%) & 2,50 \\
Não utiliza & 7,50 \\
Não produz & 37,50 \\
Não informou & 30,00 \\
\hline Total & $\mathbf{1 0 0 , 0 0}$ \\
\hline
\end{tabular}

FONTE: dados da pesquisa.

Da Tabela 11 depreende-se ser muito baixa a participação da agricultura urbana na renda das famílias do bairro de Montese, pois a prática ainda é bastante incipiente e destinada principalmente para o preparo de remédios caseiros $(10,0 \%)$, e para satisfazer pequenas necessidades de consumo. Outros $5,0 \%$ disseram comercializar alguma produção na própria casa. No Quadro 1 é apresentado os principais cultivos e criações desenvolvidas pelos entrevistados no bairro de Montese.

QUADRO 1 - Plantas e animais presentes na prática da AU no bairro de Montese.

\begin{tabular}{|c|c|c|c|}
\hline Plantas Medicinais & Hortaliças & Fruteiras & Aves \\
\hline $\begin{array}{c}\text { Pirarucu } \\
\text { (Kalanchoe pinnata) }\end{array}$ & $\begin{array}{c}\text { Alface } \\
\text { (Lactuca sativa) }\end{array}$ & $\begin{array}{c}\text { Abacaxi } \\
\text { (Ananas comosus) }\end{array}$ & \\
\hline $\begin{array}{c}\text { Babosa } \\
\text { (Aloe vera) }\end{array}$ & $\begin{array}{c}\text { Chicória } \\
\text { (Cichorium intybus) }\end{array}$ & $\begin{array}{c}\text { Açaí } \\
\text { (Euterpe oleracea Mart.) }\end{array}$ & \\
\hline $\begin{array}{c}\text { Capim santo } \\
\text { (Cymbopogon citratus) }\end{array}$ & $\begin{array}{c}\text { Cheiro verde } \\
\text { (Coriandrum sativum) }\end{array}$ & $\begin{array}{l}\text { Limão } \\
\text { (Citrus limon) }\end{array}$ & $\begin{array}{l}\text { Galinhas } \\
\text { e patos }\end{array}$ \\
\hline $\begin{array}{c}\text { Cidreira } \\
\text { (Melissa officinalis) }\end{array}$ & $\begin{array}{c}\text { Cebolinha } \\
\text { (Allium schoenoprasum) }\end{array}$ & $\begin{array}{c}\text { Cacau } \\
\text { (Theobroma cacao) }\end{array}$ & \\
\hline $\begin{array}{c}\text { Arruda } \\
\text { (Ruta graveolens) }\end{array}$ & $\begin{array}{c}\text { Jambu } \\
\text { (Acmella oleracea) }\end{array}$ & $\begin{array}{c}\text { Mamão } \\
\text { (Carica papaya) }\end{array}$ & \\
\hline
\end{tabular}

Fonte: dados da pesquisa.

Todos esses produtos são, prioritariamente, consumidos in natura e os remédios caseiros são comercializados e consumidos a partir de embalagens em garrafas e/ou in natura. Cinco moradores (12,5\%) informaram que criam galinhas e patos para o consumo próprio dos ovos e da carne. A Agricultura Urbana realizada no bairro de Montese ainda se mostra bastante incipiente, sendo a produção agrícola voltada para o autoconsumo e para produção de medicamentos naturais. 
De acordo com os entrevistados, as maiores limitações para o desenvolvimento da AU no bairro é a carência de orientação técnica e de incentivo para realizarem essa prática. Outras demandas dos entrevistados quanto às condições de infraestrutura do bairro foram: alagamentos de vias públicas e domicílios $(22,5 \%)$, saneamento básico $(22,5 \%)$, violência $(12,5 \%)$, pavimentação das ruas $(5,00 \%)$ e a interrupção no fornecimento de água distribuída pela COSAMPA (2,50\%). Essas questões, de certa forma, também, impactam na expansão da prática da agricultura urbana no bairro.

\section{CONCLUSÃO}

O desenvolvimento da agricultura urbana na área de estudo pode ser estimulado a partir de estratégias de extensão universitária, por meio da disseminação de conhecimentos técnicos e de assistência de qualidade para os interessados na comunidade. Dessa forma, pode-se contribuir para minorar o problema da pobreza no bairro, principalmente considerando o significativo peso relativo da alimentação na renda dessa comunidade.

De qualquer forma, iniciativas dessa natureza precisam ser articulada com uma ampla parceria envolvendo lideranças da comunidade, Prefeitura, Secretaria de Agricultura e Urbanismo, Igrejas, Universidades e outros atores, como forma de buscar encaminhamentos práticos para implementar e acompanhar a iniciativa de forma sistêmica.

\section{REFERÊNCIAS}

ALCANTARA, C. F. S. M.; GONTIJO, F. S. Os museus, as coisas e as comunidades: Novas percepções a partir do bairro da terra firme em Belém, Pará. Revista Eletrônica Ventilando Acervos, v. 3, n. 1, p. 92-109, nov. 2015.

BOUKHARAEVA, L. M.; CHIANCA, G. K.; MARLOIE, M.; MACHADO, A. T.; MACHADO, C. T. T. Agricultura urbana como um componente do desenvolvimento humano sustentável: Brasil, França e Rússia. Cadernos de Ciência e Tecnologia, 22(2): p. 413-425, 2005.

COSTA, M. R. T. R; HOMMA, A. K. O.; REBELLO, F. K.; SOUZA FILHO, A. P.S.; FERNANDES, G. L. C.; BALEIXE, W. Atividade agropecuária no estado do Pará. Belém: Embrapa Amazônia Oriental, 2017. (Documentos 432). Disponível em: https://www.embrapa.br/busca-de-publicacoes/-/publicacao/1073940/atividade-agro pecuaria-no-estado-do-para. Acesso em: 26 nov. 2018.

DAROLT, M. R.; LAMIENE, C.; BRANDENBURG, A.; ALENCAR, M. C. F.; ABREU, L.S. Alternative food networks and new producer-consumer relations in France and in Brazil. Ambiente \& Sociedade, vol.19, n.2, p.1-22, 2016. doi.org/10.1590/18094422ASOC121132V1922016.

DIAS, M. L.; COSTA, C. H. G.; SANTOS, J. I. N.; FERREIRA, M. R. S.; SILVA, N. R. Terra Firme: Uma reflexão sobre o atual processo de regularização fundiária. Revista Universo \& Extensão, Vol. 1, N. 1. Belém, 2013.

DUERRENBERGER, N.; WARNING, S. Corruption and education in developing countries: The role of public vs. private funding of higher education. International Journal of Educational Development, v. 62, p. 217-225. 2018. doi:10.1016/j.ijedudev.2018.05.002. 
FAO/MDS. A AUP na América Latina e no Caribe: uma realidade. Publicação distribuída no $5^{\circ}$ Fórum Urbano Mundial (Rio, 03/2010). Disponível em: $<$ http://agriculturaurbana.org.br/textos/AUPenALC-BrochureIPES_FAO-portugues_lo w.pdf>. Acesso em: Acesso em: 20 jan. 2018.

FAO/MDS. Sistematizar e analisar a implementação da Política Nacional de Agricultura Urbana e Periurbana promovida pelo Ministério de Desenvolvimento Social e Combate à Fome no Brasil com foco em regiões Metropolitanas durante o período 2004-2009. FAO/MDS, 2011. Disponível em: $<$ http://agriculturaurbana.org.br/textos/Produto_04_FAO-MDS_consolidado.pdf>. Acesso em: 20 fev. 2017.

FERREIRA, A.; GUILHERME, R.; FERREIRA, C.; OLIVEIRA M. Urban Agriculture, a tool towards more resilient urban communities?. Current Opinion in Environmental Science \& Health, 2018. doi:10.1016/j.coesh.2018.06.004.

GOUVÊA, L. "É nois" na tela: comunicação, territórios simbólicos e produção de vínculos a partir da experiência do coletivo Tela Firme na cidade de Belém (PA). Parágrafo, v. 5, n.1, p. 169-178, 2017.

LEITE JÚNIOR, D.; ALMEIDA, R. Terra Firme, um quilombo urbano em Belém. 2012. Disponível em: <https://www.cartamaior.com.br/?/Editoria/Direitos-Humanos/ Terra-Firme-um-quilombo-urbano-em-Belem/5/25093>. Acesso em: 20 jan. 2018.

MATOS, F. C.; TARGA, M. S.; BATISTA, G. T.; DIAS, N. W. Análise temporal da expansão urbana no entorno do lgarapé Tucunduba, Belém, PA, Brasil. Revista Biociências, v. 17, n. 1, 2011.

PREFEITURA MUNICIPAL DE BELÉM. Anuário Estatístico do Município de Belém. 2012. Disponível em <http://www.belem.pa.gov.br/app/c2ms/v/?id=2\& conteudo=4485 $>$. Acesso em: $21 \mathrm{dez} .2017$.

REBELLO, F. K.; HOMMA, A. K. O. História da colonização do Nordeste Paraense: reflexões para o futuro da Amazônia. Belém: EDUFRA, 2017.

REBELLO, M. G.; OLIVEIRA, C. M. L.; NOGUEIRA, A. J. S.; CORRÊA, D. B.; REBELLO, F. K. Percepção de professores sobre saúde bucal infantil. Holos, v. 05, ano 34, p. 216-230, 2018. Disponível em: <DOI: https://doi.org/10.15628/holos. 2018.7675>.

ROSA, P. P. V. Políticas públicas em agricultura urbana e periurbana no Brasil. Revista Geográfica de América Central, v. 2, n. 47, 2011.

SANTANDREU, A.; LOVO, I. C. Panorama da Agricultura Urbana e Periurbana no Brasil e Diretrizes Políticas para sua Promoção. Documento referencial geral. Belo Horizonte: REDE, IPES, n. 4, 2007.

SCHNEIDER, S.; XAVIES, L. Pluriatividade e plurirrendimentos nos estabelecimentos agropecuários do Brasil e das regiões Sul e Nordeste: uma análise a partir do Censo Agropecuário 2006. Brasília: IPEA, 2013. 77p. (Relatório de Pesquisa). 
SILVA, E. R. R.; SABLAYROLLES, M. G. P. Caracterização das Atividades de Agricultura Urbana: Um Estudo de Caso em Santarém - Pará. Cadernos de Agroecologia, [S.I.], v. 9, n. 4, fev. 2015. Disponível em: <http://aba-agroecologia. org.br/revistas/index.php/cad/article/view/16419>. Acesso em: 17 jun. 2017. 